\title{
Differential role of inhibition in habituation of two independent afferent pathways to a common motor output
}

\author{
Adam S. Bristol and Thomas J. Carew ${ }^{1}$ \\ Department of Neurobiology and Behavior, University of California, Irvine, California 92697-4550, USA
}

\begin{abstract}
Many studies of the neural mechanisms of learning have focused on habituation, a simple form of learning in which a response decrements with repeated stimulation. In the siphon-elicited siphon withdrawal reflex (S-SWR) of the marine mollusk Aplysia, the prevailing view is that homosynaptic depression of primary sensory afferents underlies short-term habituation. Here we examined whether this mechanism is also utilized in habituation of the tail-elicited siphon withdrawal reflex (T-SWR), which is triggered by an independent, polysynaptic afferent pathway that converges onto the same siphon motor neurons (MNs). By using semi-intact preparations in which tail and/or siphon input to siphon MNs could be measured, we found that repeated tail stimuli administered in the presence of a reversible conduction block of the nerves downstream of the tail sensory neurons (SNs) completely abolished the induction of habituation. Subsequent retraining revealed no evidence of savings, indicating that the tail SNs and their immediate interneuronal targets are not the locus of plasticity underlying T-SWR habituation. The networks closely associated with the siphon MNs are modulated by cholinergic inhibition. We next examined the effects of network disinhibition on S-SWR and T-SWR habituation using an Ach receptor antagonist d-tubocurarine. We found that the resulting network disinhibition disrupted T-SWR, but not S-SWR, habituation. Indeed, repeated tail stimulation in the presence of d-tubocurarine resulted in an initial enhancement in responding. Lastly, we tested whether habituation of T-SWR generalized to S-SWR and found that it did not. Collectively, these data indicate that (1) unlike S-SWR, habituation of T-SWR does not involve homosynaptic depression of SNs; and (2) the sensitivity of T-SWR habituation to network disinhibition is consistent with an interneuronal plasticity mechanism that is unique to the T-SWR circuit, since it does not alter S-SWR.
\end{abstract}

Habituation, the gradual waning of an evoked behavioral response with repeated stimulation, is a basic and universal form of learning (Thompson and Spencer 1966; Bristol and Carew 2003). Habituation has long served as a model paradigm for the study of the neural basis of learning and memory because of its relative simplicity and because of its expression in simple behaviors, such as defensive reflexes, which are often well understood in terms of their neural circuits (Horn 1967). However, despite several decades of research, a complete understanding of the neural mechanisms of habituation has not been achieved in any system.

The neural mechanisms of nonassociative learning have been examined in numerous invertebrate preparations (Abramson 1994). One system that has been extensively examined is the siphon withdrawal reflex of the marine mollusk Aplysia, in particular the siphon-elicited siphon withdrawal reflex (S-SWR). The study of Aplysia S-SWR learning offers several experimental advantages. First, the S-SWR is a simple behavior that can be easily quantified. Second, Aplysia possess a relatively simple and accessible nervous system, thus allowing for a detailed characterization of the underlying circuitry and its connectivity and synaptic plasticity. Several early studies of the cellular basis of habituation in Aplysia helped establish the prevailing view that habituation results from homosynaptic depression (an activity-dependent decrement in synaptic efficacy) of the connections between siphon sensory neurons (SNs) and siphon motor neurons (MNs) (Pinsker et al. 1969, 1970; Castellucci et al. 1970, 1978; Kupfer-

\section{'Corresponding author.}

E-mail tcarew@uci.edu; fax (949) 824-5161.

Article published online before print. Article and publication date are at http:// www.learnmem.org/cgi/doi/10.1101/lm.83405. mann et al. 1970). The results of more recent work by Hawkins and colleagues (Cohen et al. 1997; Frost et al. 1997; Antonov et al. 1999) support this view (see also Ezzeddine and Glanzman 2003). Moreover, these data are in accord with a larger body of evidence linking synaptic depression with behavioral habituation in a variety of preparations (for a review, see Christoffersen 1997).

In addition to S-SWR, defensive siphon withdrawal in Aplysia can also be evoked by stimulation of sufficient intensity virtually anywhere on the animal's body. This raises the interesting question of whether the mechanisms underlying habituation observed for S-SWR also underlie habituation resulting from activation of other afferent pathways. For example, several lines of evidence suggest that habituation of the tail-elicited siphon withdrawal (T-SWR) does not rely exclusively on homosynaptic depression of primary tail SNs onto siphon MNs. First, tail SNs do not directly synapse onto siphon MNs: Tail SNs reside in the pleural ganglia in the circumesophageal ring ganglia (Walters et al. 1983), whereas siphon MNs are located in the abdominal ganglion (Kandel 1979; Hickie and Walters 1995). Thus, strictly speaking, homosynaptic depression of SN-MN connections cannot underlie habituation of T-SWR, although an analogous mechanism might be homosynaptic depression of connections between tail SNs and their target interneurons (INTs). However, Stopfer and Carew (1996) found that repeated tail stimulation that yielded behavioral habituation of the T-SWR resulted in facilitation, not depression, of transmission between tail SN and tail MNs. Lastly, Bristol et al. (2004a) recently found that a noxious shock to one side of the body, which normally results in side-specific, lateralized sensitization of T-SWR, could also enhance the T-SWR elicited by contralateral stimulation if the con- 
tralateral side is previously habituated (contralateral dishabituation). This effect could be mimicked by applying d-tubocurarine (d-TC), an antagonist of fast inhibitory cholinergic transmission in Aplysia (Tauc and Gerschfeld 1962; Blankenship et al. 1971; Kehoe 1972; Trudeau and Castellucci 1993), to the abdominal ganglion (Bristol et al. 2004b). These results indicated that habituation of T-SWR engages one or more mechanisms among downstream INTs in the abdominal ganglion, which would be unlikely to occur if repeated tail stimulation significantly depressed tail SN output.

This study sought to directly examine and compare the mechanisms underlying habituation of the T-SWR and S-SWR, two withdrawal reflexes evoked by independent afferent pathways that converge onto a common source of motor output, the siphon MNs. We first show that blockade of neural transmission between tail sensory input and siphon motor output (using a conduction block of the pleural-abdominal connectives) abolishes the induction of T-SWR habituation. Subsequent retraining yielded habituation curves that were identical to previously untrained controls, indicating that habituation training conducted in the presence of the conduction block had no residual effect. Next, we found that network disinhibition in the abdominal ganglion using d-TC had a differential effect on habituation of the T-SWR versus S-SWR reflexes: Habituation of T-SWR was abolished by d-TC, whereas habituation of S-SWR was not affected by d-TC. Lastly, we found that habituation of T-SWR did not generalize to S-SWR. Collectively, these results indicate (1) that multiple mechanisms underlie habituation of the siphon withdrawal reflex in Aplysia, depending on which afferent pathway is engaged during training; and (2) that network inhibition is a necessary component in the induction of the decrementing process underlying T-SWR habituation.

Some of these data have appeared previously in abstract form (Bristol and Carew 2003).

\section{Results}

\section{Activation of circuits downstream from the SN synapse is required for habituation of T-SWR}

Previous results suggested that the locus of change underlying habituation of T-SWR involves INTs downstream of tail SNs (e.g., Stopfer and Carew 1996). We directly tested this hypothesis by using isotonic $\mathrm{MgCl}_{2}$ as a rapid and reversible conduction block (Walters 1987) at a segment of the pleural-abdominal connectives (P-ACs), the nerves linking tail SNs in the ring ganglia with downstream INTs and MNs in the abdominal ganglion (Bristol et al. 2004a). We reasoned that, if reduction of transmission at the tail SN synapses is the locus of change underlying T-SWR habituation, then training administered in the presence of the conduction block should have no effect on T-SWR habituation, because the block would be downstream of the site of plasticity. Conversely, if downstream INTs in the abdominal ganglion are the locus of change, then habituation training administered in the presence of the conduction block of the P-ACs should prevent habituation.

Figure 1A illustrates our experimental design. Our analysis focused on a neural correlate of SWR: the sensory-evoked complex EPSP in hyperpolarized siphon MNs (see Materials and Methods). We found that the habituation training protocol (10 taps to the dorsal tail, $10 \mathrm{sec}$ ISI, see Materials and Methods) we used previously (Bristol et al. 2004a,b) again produced significant decrement of the tail tap-evoked siphon MN EPSP (average pretest versus 10th habituation trial; $t_{(4)}=7.33, P<0.01$ ). Habituation persisted for 2-7 min (average pre-test versus $2 \mathrm{~min}$ post-test; $t_{(4)}=6.25, P<0.01$ ) (Fig. 1C). However, when the same habituation training was conducted in the presence of the $\mathrm{MgCl}_{2}$ con-
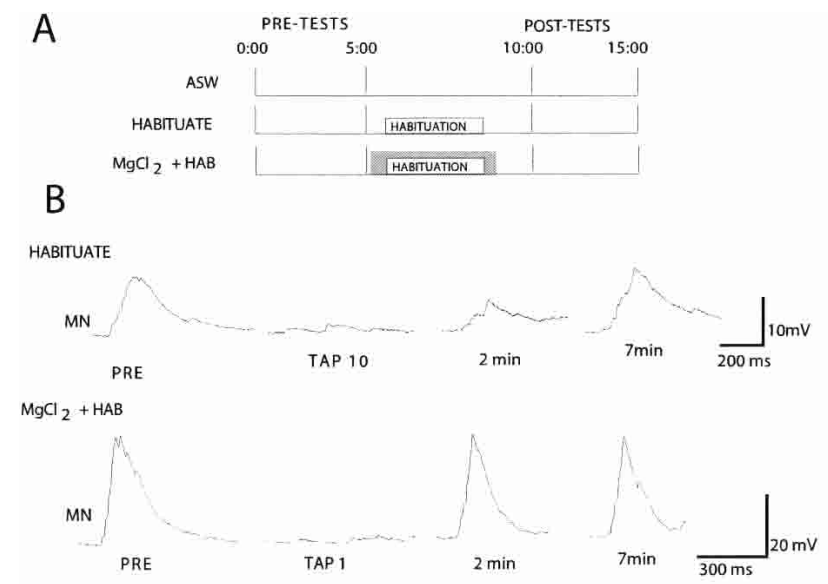

C

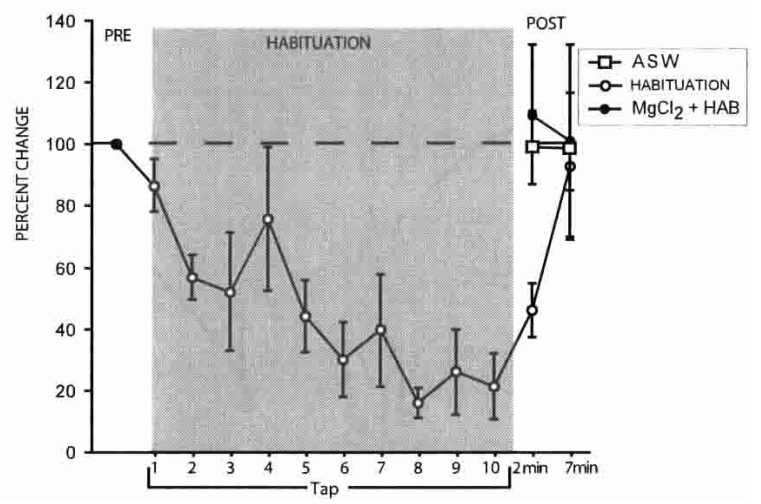

Figure 1. T-SWR habituation does not occur when training occurs in the presence of a conduction block of the P-ACs. (A) Illustration of experimental protocol. (B) Intracellular recordings from siphon MNs in two separate preparations during T-SWR habituation training conducted in ASW (top) and while conduction via the P-ACs was blocked using a $\mathrm{MgCl}_{2}$ conduction block (bottom). In ASW, the tail tap-evoked $\mathrm{MN}$ response showed substantial decrement after 10 taps, and this response recovered to baseline within $10 \mathrm{~min}$. Following training under P-AC conduction block, the MN response showed no decrement. (C) Summary data from 10 experiments showing that repeated tail stimulation decrements the tail tap-evoked response in siphon MNs (HABITUATION) and that the same training conducted in the presence of a conduction block of the $\mathrm{P}-\mathrm{ACs}\left(\mathrm{MgCl}_{2}+\mathrm{HAB}\right)$ yields responses similar to nonhabituated controls (ASW).

duction block of the P-ACs, no response decrement was observed (average pre-test versus 2 min post-test, $t_{(4)}=0.41, P=\mathrm{NS}$ ) (Fig. $1 \mathrm{C})$. This effect was not due to any reflex-enhancing effects of the $\mathrm{MgCl}_{2}$ conduction block that may have offset the habituation process since bath exchanges of $\mathrm{MgCl}_{2}$ alone had no effect on tail tap-evoked response in the siphon $\mathrm{MN}$ (average pre-test versus 2 min post-test, $t_{(4)}=0.45, \mathrm{p}=\mathrm{NS}$ ) (data not shown). Figure $1 \mathrm{~B}$ shows representative traces recorded during habituation training in the ASW control and $\mathrm{MgCl}_{2}$ block conditions. Note that the response to the first habituation trial (TAP 1) is completely abolished in the $\mathrm{MglCl}_{2}$ block condition, indicating that the conduction block was effective in eliminating tail-evoked input to siphon MNs.

It is possible that habituation training in the presence of the $\mathrm{MgCl}_{2}$ block might still induce a latent mechanism in the ring ganglia that could contribute to the decrement of T-SWR. If this were the case, then such an effect would be revealed as savings upon retraining. We tested this possibility in two separate groups of preparations (Fig. 2A). One group received two habituation training sets: the first conducted in the presence of a $\mathrm{MgCl}_{2}$ 
A

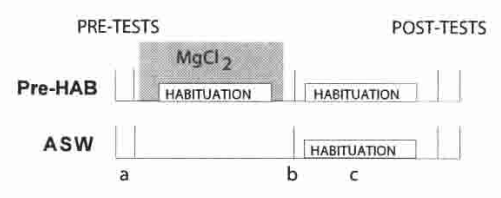

B
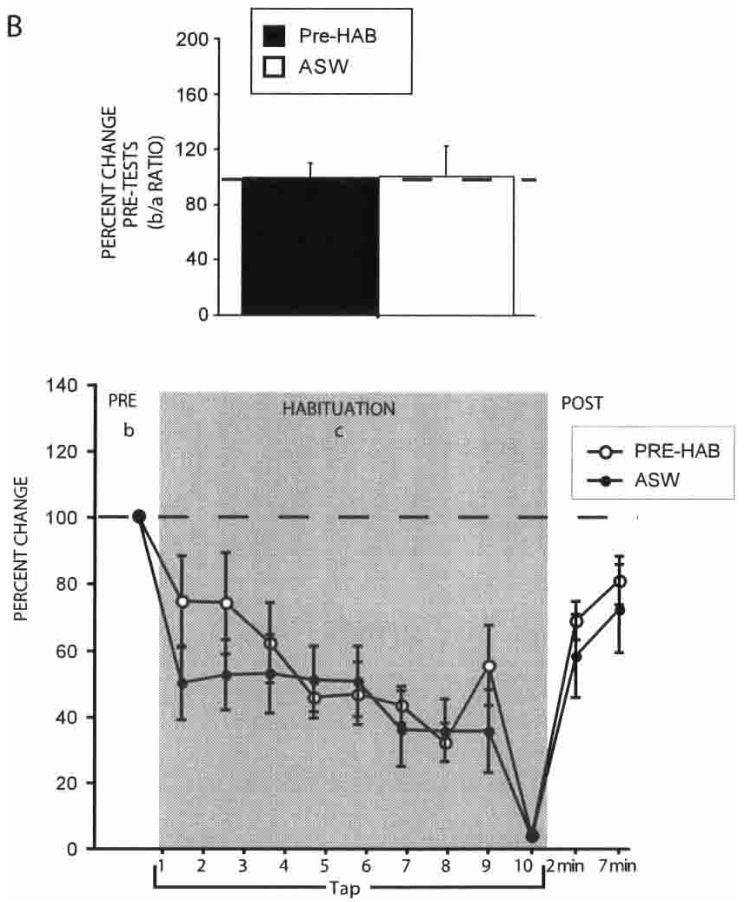

Figure 2. Lack of savings following habituation training in the presence of a conduction block of the P-ACs. (A) Illustration of experimental protocol. The letters $a, b$, and $c$ signify the initial pre-tests, the pre-tests for the second phase of training, and the habituation training, respectively. (B) Ratio of post-test response following T-SWR habituation training in the presence of $\mathrm{MgCl}_{2}$ conduction block or equivalent time point in ASW controls ( $b$ in above protocol) to initial pre-tests of tail tap-evoked MN responses ( $a$ in above protocol). Neither habituation training with the conduction block (solid bar; $\mathrm{N}=6$ ) nor time alone (open bar; $N=5$ ) changed tap-evoked $\mathrm{MN}$ response. (C) Summary of 11 experiments showing overlapping habituation and recovery curves generated by naive preps (ASW) and those previously trained under conduction block (Pre$H A B)$.

blockade of the P-ACs, the second $\sim 2$ min later in the presence of ASW (1 min after $\mathrm{MgCl}_{2}$ blockade washout). A control group underwent the same $\mathrm{MgCl}_{2}$ bath exchange treatment except that it only received the second habituation training in ASW. Two minutes post-training was the earliest possible time point to initiate the second habituation training in the $\mathrm{MgCl}_{2}$ group given the time necessary to wash out the conduction blockade (see Materials and Methods). Nonetheless, control preparations show little recovery by $2 \mathrm{~min}$ (e.g., Fig. 1C), indicating that our second habituation training was well within the temporal boundaries of any learning produced by the first training set.

As expected, the first habituation training conducted in the presence of the $\mathrm{MgCl}_{2}$ conduction block yielded no response decrement (ratio of second training pre-test to average pre-test for first training; $t_{(4)}=0.20, P=\mathrm{NS}$ ) (Fig. $2 \mathrm{~B}$, see figure caption). Upon subsequent rehabituation, we observed no evidence of savings: The acquisition curve generated by preparations trained previously in the presence of $\mathrm{MgCl}_{2}$ block was not different from that generated by control preparations trained only once in ASW
(ASW vs. PRE-HAB at 2 min post-test; $t_{(9)}=0.82, P=\mathrm{NS}$ ) (Fig. $2 \mathrm{C}$ ). Together, these data indicate that activation of circuits within the abdominal ganglion is required for the acquisition of T-SWR habituation. In addition, the observation that habituation training in the presence of $\mathrm{MgCl}_{2}$ blockade of the P-ACs results in no observable savings suggests that the mechanism underlying habituation of T-SWR resides in the abdominal ganglion.

\section{Differential effects of network disinhibition on habituation of T-SWR and S-SWR}

Since tail SNs do not project down the P-ACs to the abdominal ganglion, the site of siphon MNs (Walters et al. 1983; Marinesco and Carew 2002; Zhang et al. 2003), the basic circuit underlying T-SWR must involve polysynaptic pathways. We previously showed that bath application of the nicotinic cholinergic (nAch) blocker d-TC, which blocks fast inhibition in Aplysia (e.g., Tauc and Gerschfeld 1962; Blankenship et al. 1971; Kehoe 1972), enhances T-SWR when applied to the abdominal but not the ring ganglia (Bristol et al. 2004b). These data suggested that (1) most interneuronal processing in the T-SWR circuit resides in the abdominal ganglion, and (2) inhibitory processing normally limits the amplitude of T-SWR. S-SWR activation also recruits network inhibition: Trudeau and Castellucci (1993) found that network disinhibition using d-TC enhanced baseline S-SWR. Thus, both T-SWR and S-SWR neural circuit utilize inhibitory processing, yet only the S-SWR contains direct SN-MN connections.

Given these facts, we hypothesized that d-TC would affect habituation of T-SWR yet have little or no effect on habituation of S-SWR. We reasoned that, if the mechanism underlying habituation of T-SWR does indeed involve downstream INTs, then blocking network inhibition with d-TC might disrupt INT processing underlying habituation. In contrast, if habituation of SSWR is due primarily to homosynaptic depression of SN-MN synapses, then d-TC should have no effect, either because d-TC does not alter excitatory transmission (at the appropriate concentration) (Carpenter et al. 1977; Trudeau and Castellucci 1993) or because SN-INT synapses should also be depressed, thus decreasing synaptic drive into the S-SWR circuit.

We tested this prediction by habituating T-SWR and S-SWR reflexes in the presence or absence of $100 \mu \mathrm{M}$ d-TC (Fig. 3A). This concentration of d-TC has been shown previously to block inhibitory transmission while sparing excitatory transmission (Trudeau and Castellucci 1993). Figure 3B shows the effect of bath-applied d-TC on baseline tail- and siphon-evoked responses in siphon MNs. Consistent with previous reports (Trudeau and Castellucci 1993; Bristol et al. 2004b), both T-SWR and S-SWR

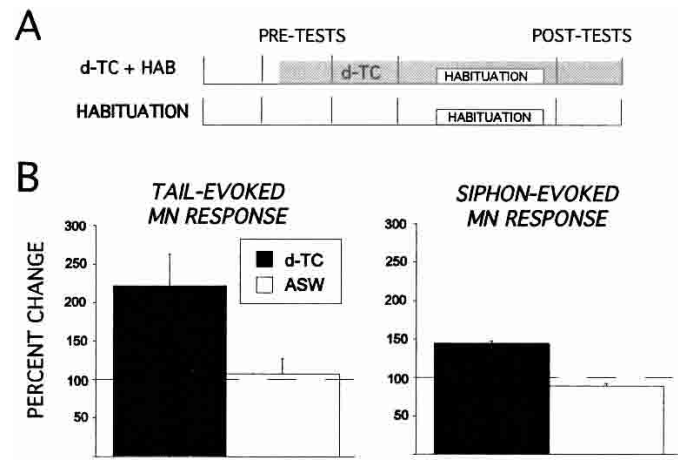

Figure 3. Network disinhibition enhances baseline T-SWR and S-SWR. (A) Illustration of experimental protocol. (B) Histograms depicting the enhancement of baseline T-SWR (left, $\mathrm{N}=5$ ) and S-SWR (right, $\mathrm{N}=5$ ) following $100 \mu \mathrm{M} \mathrm{d}$-TC exposure. ASW treatment alone resulted in no change in baseline T-SWR $(\mathrm{N}=5)$ or S-SWR $(\mathrm{N}=5)$.

\section{Learning \& Memory}

www.learnmem.org 
were enhanced following 10 min exposure to d-TC (T-SWR average increase $=221 \% ; t_{(8)}=2.50, P<0.05$; S-SWR average increase $148 \% ; t_{(7)}=8.36, P<0.05$ ) (Fig. $3 \mathrm{~B}$ ). Thus, the d-TC enhancement of the baseline response illustrated in Figure 3 served as a positive control for drug efficacy in each experiment. d-TC had a significantly greater enhancing effect on T-SWR compared with S-SWR $\left(t_{(9)}=16.08, P<0.05\right)$, although this effect is likely due to the generally higher baseline response elicited by siphon stimulation. We next examined the effects of habituation training from the d-TC-enhanced baseline.

The effects of d-TC on habituation of T-SWR are shown in Figure 4. In contrast to control preparations trained in ASW, habituation training conducted in the presence of d-TC resulted in no appreciable response decrement (ASW versus d-TC at 10th habituation trial; $t_{(12)}=3.03, P<0.05$ ) (Fig. 4B). Moreover, in three of five experiments repeated stimulation during the early portion of the habituation training in d-TC resulted in a increase in responding. Figure $4 \mathrm{~A} 2$ illustrates one of these experiments. In addition, the 2-min and 7-min post-tests were marginally enhanced following habituation training in the presence of d-TC.

The effects of d-TC on habituation of S-SWR are shown in Figure 5. Whereas d-TC exposure disrupted habituation of T-SWR (Fig. 4), the same treatment had no effect on habituation of S-

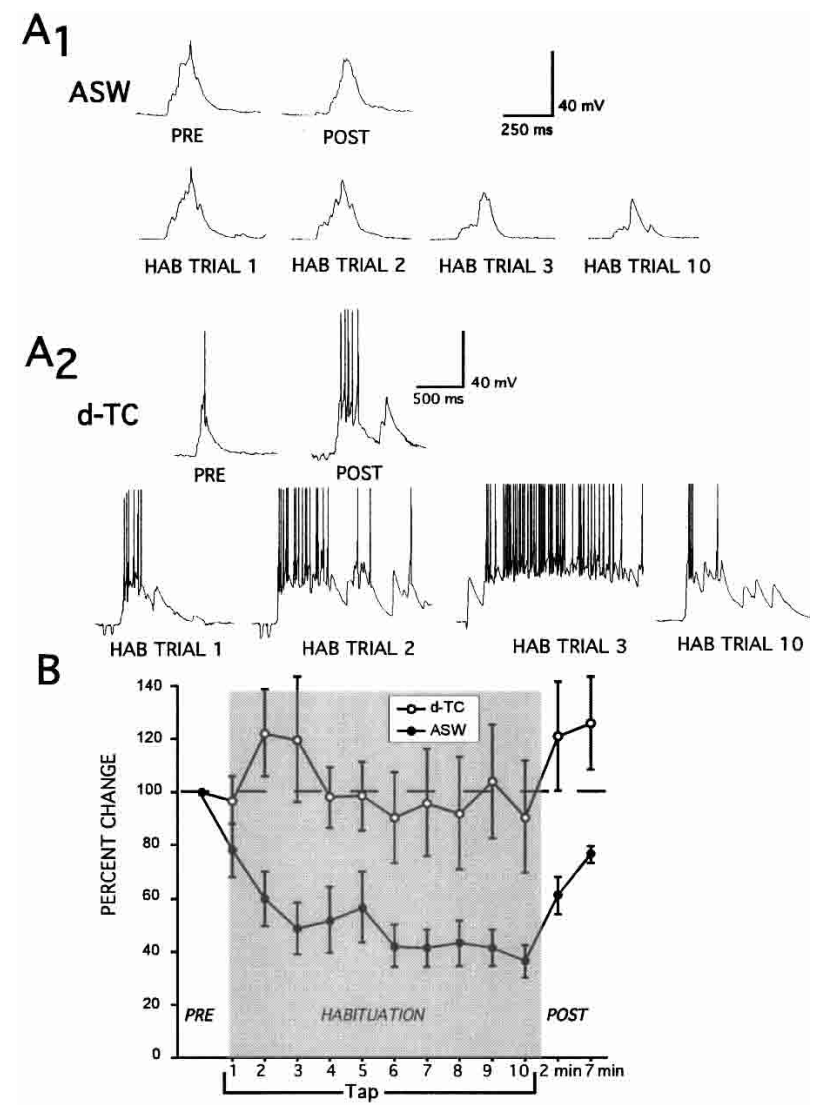

Figure 4. Network disinhibition disrupts habituation of T-SWR. $(A)$ Intracellular recording from siphon MNs during T-SWR habituation experiments under conditions of ASW control (A1) and d-TC disinhibition (A2). (A1) In ASW, the tail tap-evoked MN responses did not change across pre-tests. The response diminished across habituation trials. (A2) The tap-evoked MN response increased after addition of $100 \mu \mathrm{M} \mathrm{d}$-TC to the bath. In addition, the response did not diminish across habituation trials. In this case, d-TC resulted in a marked increase in the tap-evoked response during the first three habituation trials. (B) Summary of 14 experiments showing the disruption of T-SWR habituation by d-TC disinhibition.
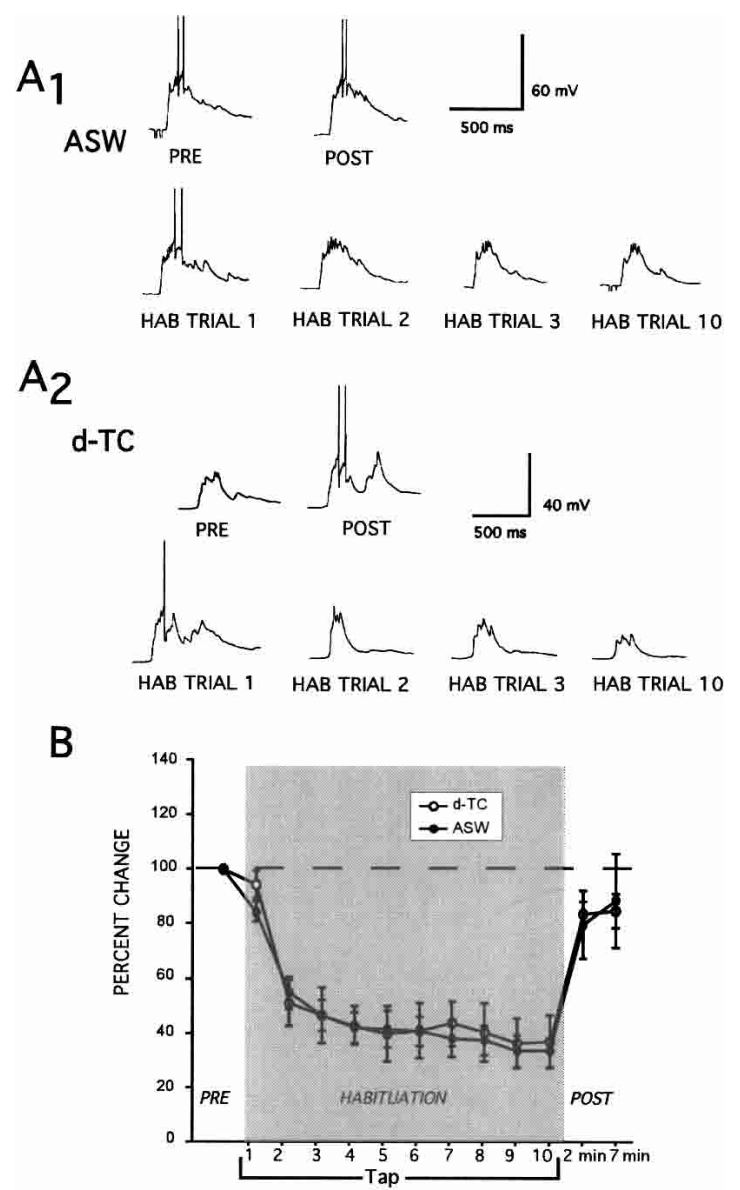

Figure 5. Network disinhibition does not affect habituation of S-SWR. (A) Intracellular recording from siphon MNs during S-SWR habituation experiments under conditions of ASW control $(A 1)$ and d-TC disinhibition (A2). (A1) In ASW, the siphon tap-evoked MN responses did not change across pre-tests. The response diminished across habituation trials. (A2) The tap-evoked $\mathrm{MN}$ response increased after addition of $100 \mu \mathrm{M} \mathrm{d}$-TC to the bath. However, the response similarly diminished across habituation trials. (B) Summary of 10 experiments showing no effect of d-TC disinhibition on habituation of S-SWR.

SWR. Despite the enhanced baseline, the habituation curve generated by d-TC-treated preparations is indistinguishable from that produced by the ASW-treated group (ASW versus d-TC at 10th habituation trial $t_{(8)}=0.35, P=\mathrm{NS}$ ) (Fig. 5B). The traces illustrated in Figure 5A show that the siphon-evoked response in the siphon MN gradually declined with repeated stimulation in the presence of d-TC. These results indicate that network disinhibition induced by d-TC affects habituation of T-SWR but not that of S-SWR.

We were also interested in whether the effects of d-TC on T-SWR habituation were specific to the cholinergic system. In several experiments, we examined habituation of T-SWR in the presence of picrotoxin, a selective $\mathrm{GABA}_{\mathrm{A}}$ antagonist effective in Aplysia (e.g., Jing et al. 2003). However, even up to bath concentrations as high as $1 \mathrm{mM}$, picrotoxin had no effect on the basal T-SWR (data not shown). The inability of picrotoxin to modify the tail tap-evoked $\mathrm{MN}$ response is perhaps not surprising given the lack of GABA-immunopositive cells in the abdominal ganglion (Diaz-Rios et al. 1999).

\section{T-SWR habituation does not generalize to S-SWR}

The neural circuit underlying the S-SWR has been relatively wellcharacterized (e.g., Cleary et al. 1995; Frost and Kandel 1995). 


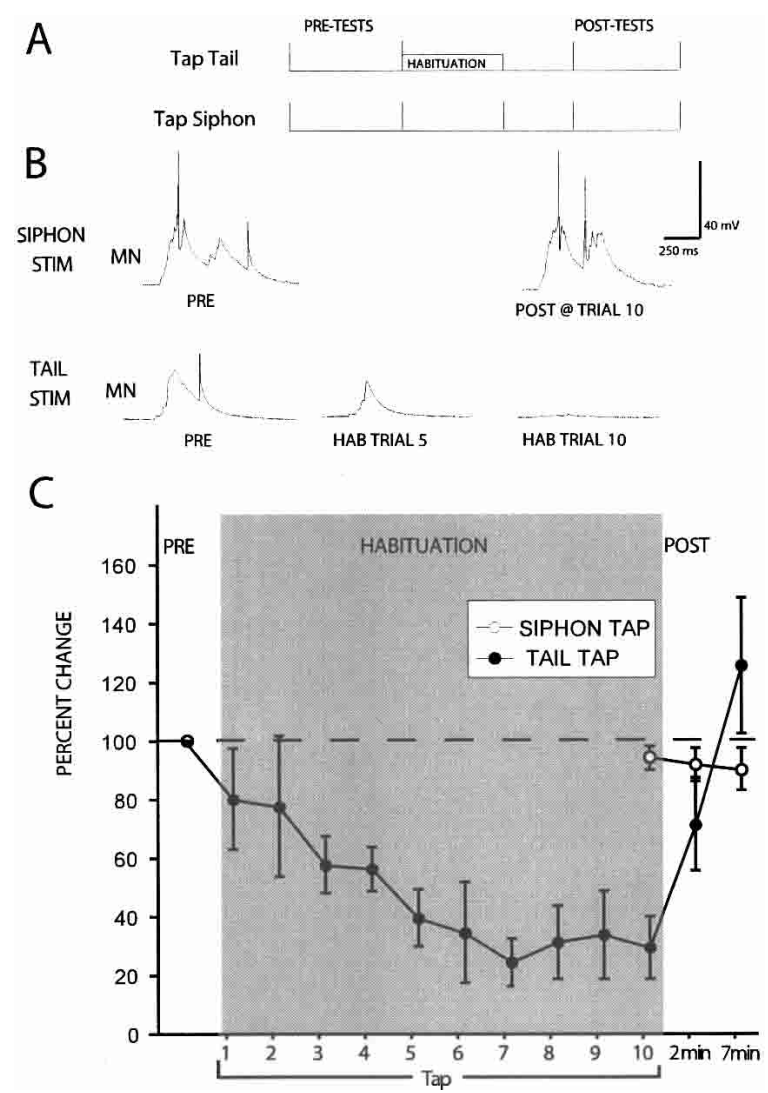

Figure 6. Habituation of T-SWR does not generalize to S-SWR. (A) IIlustration of experimental protocol. (B) Intracellular recordings from a single siphon $\mathrm{MN}$ in a preparation with which $\mathrm{MN}$ responses to both siphon (top) and tail (bottom) could be recorded. The MN response to siphon tap was unaltered by habituation of the T-SWR. (C) Summary data from five experiments showing that habituation of the T-SWR does not affect the S-SWR.

Several of the previously identified inhibitory and excitatory abdominal INTs involved in the S-SWR are also sensitive to tail stimulation (e.g., L29, L28, and L30), suggesting that these INTs may be part of the circuit mediating T-SWR. Thus, we attempted to localize the plasticity mechanism underlying habituation of T-SWR, by testing whether habituation of T-SWR generalizes to S-SWR. If so, then at least some of the T-SWR circuit elements underlying habituation would appear to be shared with the SSWR circuitry, thereby identifying possible candidate INTs in which inhibition might be exerted during T-SWR habituation.

To examine this question, we used a semi-intact preparation in which both the tail and siphon were retained. Following two baseline tests of both tail- and siphon-evoked responses in the same siphon MN, we habituated the T-SWR and examined S-SWR and T-SWR responses (Fig. 6A). Importantly, we added an additional test of S-SWR immediately after the 10th habituation stimulus (when T-SWR habituation is greatest) in order to maximize our chances of observing generalization. However, as shown in Figure 6, B and C, we found no evidence of generalization: Habituation of T-SWR had no effect on the S-SWR tested immediately after the 10th habituation stimulus or when tested again at 2 min post-training (T-SWR versus S-SWR at 10th habituation trial, $t_{(6)}=4.34, P<0.05$ ) (Fig. 6C). The raw traces shown in Figure 6B illustrate the full MN response to siphon tap immediately following the last habituation trial administered to the tail. The failure of T-SWR habituation to generalize to the
S-SWR strongly suggests that those cells involved in the process underlying T-SWR habituation are unique to the T-SWR circuit.

\section{Discussion}

In this study we examined the mechanisms underlying habituation of two defensive reflexes in the marine mollusk Aplysia (SSWR and T-SWR) that utilize different afferent pathways yet converge upon a common motor output. Considerable previous research has pointed to a predominant role for homosynaptic depression of primary SNs as the mechanism underlying habituation of S-SWR (e.g., Cohen et al. 1997). However, several findings suggest that the T-SWR, which also undergoes habituation, does not share the same mechanism (e.g., Stopfer and Carew 1996; Bristol et al. 2004a). In the present study, we first examined whether habituation of T-SWR relies on homosynaptic depression of primary SN synapses by conducting habituation training in the presence of a rapid, reversible conduction block of the P-ACs, the connectives linking the tail SNs with downstream INTs and siphon MNs. When we did so, we found that repeated tail stimulation resulted in no decrement of T-SWR. Furthermore, the habituation rates during the retraining of those preparations previously stimulated under the $\mathrm{MgCl}_{2}$ conduction block were no different from those generated by preparations habituated for the first time, indicating no residual savings from the initial training. We also found that disinhibiting the central nervous system (CNS) by bath applying the nAch inhibitor d-TC, while enhancing the baseline of both responses, disrupted habituation of T-SWR yet had no effect on habituation of S-SWR. Lastly, we found that habituation of the T-SWR does not generalize to the S-SWR.

Collectively, our data support a model in which a plasticity mechanism downstream of tail SNs accounts for habituation of T-SWR, whereas decrement of SN-MN connections accounts for habituation of S-SWR. The diagram in Figure 7 presents a model circuit containing S-SWR and T-SWR pathways and the relative positioning of the two hypothetical habituation mechanisms. In addition, the model highlights the minimal set of polysynaptic pathways to account for the effects of d-TC on reflex amplitude. As shown in Figure 7, the S-SWR pathway consists of direct connections between siphon SNs and siphon MNs as well as a feedforward excitatory pathway through an INT. Furthermore, the excitatory INT in this circuit forms a recurrent inhibitory loop an inhibitory INT, conceptually similar to a real microcircuit in the S-SWR pathway between the excitatory L29s and the inhibitory L30s (Fischer and Carew 1993, 1995; Frost and Kandel 1995). The T-SWR pathway (Fig. 7, top) does not contain direct SN-MN connections, but only polysynaptic, feed-forward excitation of siphon MNs. Similar to the S-SWR, the T-SWR network also con-

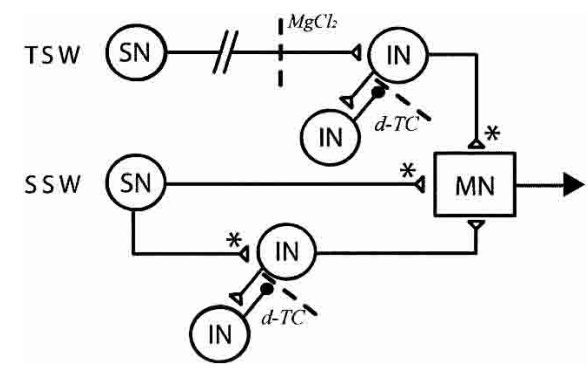

Figure 7. Model circuits depicting possible mechanisms of habituation in the T-SWR and S-SWR. Hypothesized neural circuits underlying T-SWR (top) and S-SWR (bottom). The S-SWR circuit consists of both monosynaptic and polysynaptic components. Activity-dependent, homosynaptic depression occurs at synapses marked with asterisks (*). Sites of blockade in our experiments are indicated by dashed lines. 
tains a recurrent inhibitory loop involving a subset of INTs. As indicated by asterisks $\left({ }^{*}\right)$, the model posits that the output of siphon SNs can undergo homosynaptic depression during habituation training, whereas the tail SNs do not (Stopfer and Carew 1996). Instead, in the T-SWR pathway depression occurs at the INTs making contacts onto siphon MNs, which would be consistent with the downstream location of the T-SWR habituation mechanism. We should note that, at present, there is no direct evidence for a particular INT-MN synapse as the site of depression. Since known INT-MN connections in the SWR (e.g., L34 and L29 - MNs) show little propensity for homosynaptic depression (Cleary et al. 1995; Frost and Kandel 1995; A.S. Bristol and T.J. Carew, unpubl.), it is possible that the habituationrelated depression occurs at an INT-INT connection upstream of motor output but downstream of SN-INT connections in the T-TSWR circuit.

This model circuit structure is able to account for our results in the following way: First, for the T-SWR, the failure to habituate in the presence of a conduction block occurs because the block is downstream of tail SNs but upstream of the activity-dependent plasticity mechanism $\left(^{*}\right)$. Thus, repeated tail sensory input fails to reach the INT circuit and therefore does not engage the depression mechanism. Tail SNs do not project to the abdominal ganglion, implying that a second-order INT must convey sensory information to the abdominal ganglion. The identity of this (or these) INT(s) is unknown. It is possible that the abdominal L29 INTs, of which there are subtypes that are preferentially activated by head and tail input (Fischer and Carew 1995), could mediate this information transfer since physiological (Cleary and Byrne 1993) and anatomical results (A.S. Bristol and T.J. Carew, unpubl.) indicate that they project up the P-ACs to the pleural ganglia. Second, according to the model, network disinhibition by d-TC affects the baseline amplitude of both S-SWR and T-SWR because each is regulated, in part, by a recurrent inhibitory circuit (Fig. 7). Thus, blocking the recurrent (presumably cholinergic) inhibition relieves the excitatory INT and increases reflex amplitude. However, d-TC differentially affects T-SWR habituation because the recurrent inhibition regulates the activity at the site of plasticity necessary for habituation; removal of this inhibition not only increases reflex amplitude but also allows tail-driven input to activate the excitatory INT into a spiking range (i.e., number or frequency of action potentials) outside that required for the induction of homosynaptic depression. Thus, we posit that a level of inhibitory processing in the T-SWR circuit is necessary for normal response decrement, for example, by acting as a brake on INT firing frequency and permitting the induction of depression in the circuit. Without such an inhibitory brake, repeated stimulation can facilitate the tail-evoked response, perhaps by favoring the induction of activity-dependent forms of synaptic enhancement (Fig. 4A2).

Siphon stimulation also recruits inhibitory processing (Figs. 3, 7, see also Trudeau and Castellucci 1993). Indeed, Srivatsan and Peretz (1996) noted a pronounced suppression of the S-SWR in intact animals injected with either the acetylcholinesterase antagonist BW284c51 or cholinergic agonist carbachol, both of which increase cholinergic inhibition. Despite this suppression, the response still showed additional decrement with habituation training. Although these results likely involved both central and peripheral drug effects, they are consistent with the model shown in Figure 7, for an increase in cholinergic inhibition would suppress the polysynaptic pathway in the S-SWR circuit yet would not affect the efficacy or plasticity of the monosynaptic component. Lastly, our model proposes that habituation of the T-TSWR relies on plasticity at an INT site that is not shared by the S-SWR circuit, in accord with our finding that habituation of T-SWR does not generalize to S-SWR.

\section{Inhibitory processes in habituation}

Early theoretical accounts of habituation emphasized two possible means of generating response decrement: a decrease in excitatory processes or an increase in inhibitory processes. Many early conceptions of habituation were based on increases in inhibition (Humphrey 1933; Harris 1943; Thorpe 1963). However, most contemporary cellular models of habituation, based on physiological analyses in both vertebrates (e.g., Thompson and Spencer 1966; Glanzman et al. 1972) and invertebrates (e.g., Krasne 1969; Castellucci et al. 1970), have emphasized decreases in excitation intrinsic to a reflex circuit. However, there are examples of extrinsic mechanisms of habituation, in which response decrement involves increases in inhibitory modulation. For example, whereas homosynaptic depression of presynaptic sensory cells has been found to contribute to habituation of the crayfish tail-flip reflex (e.g., Zucker 1972), Krasne and Teshiba (1995) found that an increase in tonic descending inhibition acts as an additional mechanism of habituation in that system.

Here we found that blocking network inhibition with d-TC disrupted the normal response decrement during T-SWR habituation training (Fig. 4). Our model shown in Figure 7 envisions network inhibition as a necessary cofactor in the decrementing process, without which the habituation-related homosynaptic depression of interneuronal pathways cannot be induced. This finding is also consistent with other alternatives, in particular the notion that an increase in inhibition underlies T-SWR habituation. It may be that inhibitory INTs (e.g., L30s or L16) (Hawkins 1981; Hawkins et al. 1981) are potentiated by repeated tail stimulation. To be consistent with our generalization results, this possibility would require that the inhibitory INT have a receptive field restricted to, or preferentially activated by, the tail and not the siphon. L16 indeed shows this receptive field pattern (Wright and Carew 1995), but our preliminary analyses indicate that the mild tactile stimulus we used does not activate L16 (A.S. Bristol and T.J. Carew, unpubl.). An alternative possibility is that treatment with d-TC disinhibited a facilitatory heterosynaptic mechanism that opposed the decrementing mechanism, as described for dual process models of dishabituation (e.g., Groves and Thompson 1970). As shown in Figure 4, d-TC caused facilitation of the tail-evoked $\mathrm{MN}$ response during the first few habituation trials that subsided over the course of the habituation session. This suggests that facilitatory process is activated in parallel with the decrementing process; if d-TC simply blocked the decrementing process, then one would expect no change in the tail-evoked response across habituation trials. As we indicated above, the facilitation that occurs under d-TC disinhibition could be due to the induction of activity-dependent forms of synaptic enhancement (e.g., post-tetanic potentiation) or to the activation of heterosynaptic plasticity induced by modulatory INT.

At present, we cannot discriminate between these alternative possibilities. We favor homosynaptic depression of interneuronal pathways in our model because it is more parsimonious to posit the same plasticity occurring at different loci than to posit the operation of two entirely different mechanisms. While we posit that the homosynaptic depression in the T-SWR network is conditional on the presence of effective inhibitory processing, we do differentiate between the two mechanisms of synaptic decrement; if siphon SNs exhibited a sufficient increase in excitability, homosynaptic depression might be blocked as well. Moreover, as shown in Figure 6, we found that habituation of T-SWR did not generalize to S-SWR, which one would not predict if repeated tail stimulation increased network inhibition, given the number of INTs known to be shared between the two circuits. However, additional studies will be necessary to resolve this issue, perhaps by employing either the acetylcholinesterase antagonist BW284c51 or the cholinergic agonist carbachol (e.g., Srivatsan 
and Peretz 1996). If T-SWR habituation is due to an increase in inhibition, but not homosynaptic depression, then these compounds would be expected to enhance or preclude T-SWR habituation.

Lukowiak (1978) reported that picrotoxin perfused over the abdominal ganglion both enhanced baseline siphon-elicited gill withdrawal and prevented habituation. As mentioned in the Results, we observed no effect of picrotoxin on T-SWR, even when millimolar concentrations were used. We are not sure how to reconcile these conflicting observations, considering that recent immunohistochemical analyses revealed no GABA-positive somata in the abdominal ganglion, and only a single prominent descending process in the P-ACs (Diaz-Rios et al. 1999). As T-SWR and gill withdrawal utilize distinct MNs (Koester and Kandel 1977; Hickie and Walters 1995), it is possible that this sparse GABAergic projection is specifically targeted to the gill withdrawal circuit.

The fact that we did not observe any evidence for GABAergic modulation of T-SWR strengthens the notion that cholinergic INTs regulate reflex amplitude, although d-TC may alter the responses of other transmitter receptors at higher concentrations (Carpenter et al. 1977). Stronger evidence that cholinergic INTs are specifically involved would come from paired anatomical and physiological demonstrations of transmitter content and regulation of T-SWR amplitude. Early studies by Giller Jr. and Schwartz (1971a,b) identified cells in the abdominal ganglion containing acetylcholinesterase and for the synthetic enzyme choline acetyltransferase, although many of these cells were uncharacterized. Possible candidate neurons include L16 and L30s (Hawkins 1981; Hawkins et al. 1981), both of which are tail-responsive inhibitory INTs. L16 is cholinergic (Segal and Koester 1982), and the L30s are thought to be so as well (R. Calin-Jageman and T.M. Fischer, pers. comm.).

\section{Mechanisms of habituation in Aplysia}

It is interesting that even seemingly simple forms of learning rely on multiple neuronal mechanisms. For example, long-term SSWR habituation is believed to involve both depressed synaptic transmission between SNs and MNs (e.g., Castellucci et al. 1978) and a reduction in the number of synaptic contacts made by SNs onto MNs (Bailey and Chen 1983, 1989). Recent work from Ezzeddine and Glanzman (2003) suggest that post-synaptic process in siphon MNs also occur in long-term habituation. Thus, it appears that multiple mechanisms maintain S-SWR habituation across a range of temporal domains.

Our data indicate that different mechanisms underlie habituation of two related response systems, the T-SWR and S-SWR. Our finding that d-TC does not alter S-SWR habituation is consistent with the notion that homosynaptic depression underlies S-SWR habituation (Cohen et al. 1997; Frost et al. 1997; Antonov et al. 1999). Our model is consistent with the notion that habituation of T-SWR does not differ in the basic plasticity mechanism (synaptic depression) but rather in its placement within the reflex neural circuit. The plasticity underlying T-SWR habituation appears to be contained within the polysynaptic pathways downstream of tail SNs, thus making it susceptible to disruption by disinhibitory modulation.

In conclusion, the dual reflex system that we have analyzed now permits direct assessment of the hypothesis that a common mechanism for habituation, homosynaptic depression, can reside at different sites within overlapping neuronal networks subserving different reflex pathways. If this is the case, then homosynaptic depression may represent a highly conserved cellular strategy to implement a highly conserved form of learning, habituation. The exact placement of this plasticity mechanism within a particular network may be governed by additional factors, such as the complexity of the circuit and the necessity for integration with multiple sources of input to the network.

\section{Materials and Methods}

\section{Subjects}

Adult Aplysia californica (100-300 g) were acquired commercially (Marinus; Marine Resource and Educational Products) and housed individually in a 600-L aquarium with continuously circulating artificial sea water (ASW; Instant Ocean, Aquarium Systems) at $\sim 15^{\circ} \mathrm{C}$. Animals were fed dried seaweed twice weekly.

\section{Experimental preparations}

Three kinds of preparations were used in this study: the tail alone preparation, the siphon preparation, and the tail/siphon preparation. Each of these preparations was "semi-intact," meaning that Aplysia were dissected such that peripheral structures were left connected to the CNS. All animals were first anesthetized with an injection of isotonic $\mathrm{MgCl}_{2}$ into the body cavity. For the tail-alone preparation, the tail and CNS were excised while preserving their connection via the P9 nerves. For the siphon alone preparation, all peripheral nerves were cut except the siphon nerve, which innervates the siphon and mantle shelf, and the siphon and CNS were dissected. For the tail/siphon preparation, both the tail and siphon were retained. In all cases, the CNS (abdominal ganglion and ring ganglia) and remaining peripheral structures were excised from the animal and transferred to a recording dish coated with silicone elastomer (Sylgard, DowCorning). The abdominal ganglion was briefly fixed (30-sec immersion in $0.04 \%$ gluteraldehyde) to facilitate desheathing and to prevent contractions of the connective tissue. The recording dish was fit with either two or three separate chambers: one contained the CNS, the others contained either the tail or siphon. The peripheral nerves were passed between the chambers via a small slit in the separating barrier. The slit was then sealed using petroleum jelly to improve electrical isolation and to allow for independent manipulation of bath levels. The tail and/or siphon was pinned dorsal side up and cannulated for continuous perfusion of room temperature ASW $30 \mathrm{~min}$ prior to and throughout the experiment.

\section{Procedures}

The left abdominal hemiganglion was desheathed in a 1:1 mixture of ASW and $\mathrm{MgCl}_{2}$ to prevent synaptic transmission. Throughout the experiment, ganglia were continuously perfused with ASW at room temperature $\left(20^{\circ} \mathrm{C}\right)$ at approximately $6 \mathrm{~mL} /$ min and illuminated from below through a dark field condenser. Ganglia were visualized with a Zeiss dissecting microscope fitted with a recording stage. Standard intracellular recording techniques were used. Neurons were impaled with glass microelectrodes (resistance 6-15 M 2 ) filled with $3 \mathrm{M} \mathrm{KCl}$. Electrical potentials were amplified on an Axoclamp 2B (Axon Instruments). Data analysis was conducted using a POWERLAB data acquisition unit (ADInstruments) and accompanying Chart 3.6 software.

Siphon MNs were identified by their size, location, and their spontaneous activity (Hickie and Walters 1995; Belkin and Abrams 1998). Following impalement, they were hyperpolarized to approximately $-75 \mathrm{mV}$ to reduce spiking. LFS class siphon MNs, which are dedicated to defensive siphon withdrawal (Hickie and Walters 1995), were used in this study. The tail and siphon were stimulated using stimulator-driven "tappers" (blunted low-gauge needles attached to a servo-mechanism activated by an electrical relay) to deliver a brief $(25-50 \mathrm{msec})$ tactile stimuli to the mid-line of the dorsal tail or the base of the siphon. The tapper stimuli were of weak to mild intensity $\left(1.0 \mathrm{~g} / \mathrm{mm}^{2}\right)$, yet still in an intensity range capable of eliciting SWR (e.g., Byrne et al. 1978). The area underneath the initial $500 \mathrm{msec}$ of the evoked complex EPSP in the MN was integrated $(\Delta \mathrm{mV} \cdot \mathrm{sec})$ for a quantitative measure of the net activation. Because siphon MNs are spontaneously active at rest, the evoked complex EPSP pro- 
vides sensitive measure of changes in both the amplitude and duration of the evoked response. The 500-msec integration time was chosen because it adequately encompasses the duration of the evoked MN response elicited by tail tap. In some cases, d-TCinduced disinhibition caused the evoked response to be of a considerably longer duration. In these cases, the portion of the complex EPSP that was not included in the $500-\mathrm{msec}$ integration window was excluded, and the extent of MN activation was somewhat underestimated. Occasionally, the post-synaptic MN hyperpolarization was insufficient to completely prevent spiking during stimulation (likely due to the afferent input being too great to suppress all spikes). Therefore, spikes were included in analyses of MN activation if they occurred within $500 \mathrm{msec}$ of activation.

\section{Experimental protocol}

We chose a habituation protocol that optimized response decrement lasting between 5 and $10 \mathrm{~min}$. For all experiments, the standard habituation training consisted of 10 taps delivered at a 10-sec ISI. At least two pre-tests of tap-evoked MN responses were taken at $5 \mathrm{~min}$ ITI prior to habituation training. Post-tests of evoked $\mathrm{MN}$ responses were taken at 2 and 7 min following training. Generally, the habituation training was initiated at a time after the last pretest such that the first post-test $(2 \mathrm{~min})$ would maintain a consistent 5-min ITI protocol. The exception to this occurred in the "savings" experiments, wherein the first post-test ( $2 \mathrm{~min}$ ) also served as the first stimulus of the second habituation training.

Figure 1 depicts the design of the experiments examining the effects of reversible inactivation of the P-ACs on T-SWR habituation. Following two pre-tests of tail tap-evoked MN activation, isotonic $\mathrm{MgCl}_{2}$ was hand-pipetted into a small isolated chamber containing only a short segment of the P-ACs $(\sim 2 \mathrm{~cm})$ approximately halfway between the ring and abdominal ganglia. Thus, only a short segment of the P-ACs was exposed to $\mathrm{MgCl}_{2}$. Nerve conduction was reinstated by exchanging ASW for $\mathrm{MgCl}_{2}$. Initial pilot experiments determined that complete wash-in or wash-out of the conduction block in this way takes $<45 \mathrm{sec}$. If, however, the $\mathrm{MN}$ response to the first tap of the habituation training was not abolished (indicating an incomplete conduction block), the experiment was discontinued. This occurred in only two of 18 experiments. Immediately following the last habituation stimulus (trial 10), ASW was replaced into the P-AC chamber. Control experiments utilized the same pattern of $\mathrm{MgCl}_{2}$ wash-in and wash-out, but with no habituation training. Savings experiments followed the same protocol except that the 2-min post-test following the initial habituation training served as the first stimulus in the second habituation. Two additional posttests were administered at 2 and 7 min following the second habituation training.

The design of experiments examining the effects of network disinhibition on T-SWR and S-SWR habituation is shown in Figure 3. An initial two pre-tests were taken to serve as baseline measures of tap-evoked input into the MN. Immediately following, $40 \mathrm{~mL}$ of $100 \mu \mathrm{M}$ d-TC (ICN Biochemicals) was perfused into chamber containing the CNS bath. The exchange of media bathing the CNS was stopped after d-TC perfusion ( $\sim 6 \mathrm{~min})$, and the drug was left in static bath for the duration of the experiment. Two additional pre-tests were taken in the presence of d-TC were followed by a habituation training. Post-tests were conducted at 2 min and 7 min following training.

Experiments examining the generalization of T-SWR habituation utilized the siphon/tail preparation. These experiments followed the same protocols as above, except that tests of siphonevoked MN responses were taken each pre-test and post-test as well as an additional test $10 \mathrm{sec}$ after the last T-SWR habituation stimulus.

\section{Data analysis}

Summary data are presented graphically as mean percentage change from average baseline (average of two pre-tests) \pm SEM. Differences between means were analyzed using ANOVAs and paired $t$ tests with an alpha level of 0.05 (two-tailed) adopted for all tests of statistical significance.

\section{Acknowledgments}

We thank Dr. Stéphane Marinesco for his helpful criticisms throughout the project. A.S.B. is now at the Department of Neurobiology, Stanford University. This work was supported by a National Institute of Mental Health Grant RO1 MH-14-1083 and a National Science Foundation Grant IBN-0049013 to T.J.C.

\section{References}

Abramson, C.I. 1994. A primer of invertebrate learning: A behavioral perspective. American Psychological Association, Washington, DC.

Antonov, I., Kandel, E.R., and Hawkins, R.D. 1999. The contribution of facilitation of monosynaptic PSPs to dishabituation and sensitization of the Aplysia siphon withdrawal reflex. J. Neurosci. 19: $10438-10450$

Bailey, C.H. and Chen, M. 1983. Morphological basis of long-term habituation and sensitization in Aplysia. Science 220: $91-93$ . 1989. Structural plasticity at identified synapses during long-term memory in Aplysia. J. Neurobiol. 20: 356-372.

Belkin, K.J. and Abrams, T.W. 1998. The effect of the neuropeptide FMRFamide on Aplysia californica siphon motoneurons involves multiple ionic currents that vary seasonally. J. Exp. Biol. 201: 2225-2234.

Blankenship, J.E., Wachtel, H., and Kandel, E.R. 1971. Ionic mechanisms of excitatory, inhibitory, and dual synaptic actions mediated by an identified interneuron in the abdominal ganglion of Aplysia. J. Neurophysiol. 34: 76-92.

Bristol, A.S. and Carew, T.J. 2003. The role of polysynaptic pathways in habituation of the tail-elicited siphon withdrawal in Aplysia. Soc. Neurosci. Abstr. 29: 520.3.

Bristol, A.S., Marinesco, S., and Carew, T.J. 2004a. Neural circuit of tail-elicited siphon withdrawal in Aplysia, II: Role of gated inhibition in differential lateralization of sensitization and dishabituation. $J$. Neurophysiol. 91: 678-692.

Bristol, A.S., Sutton, M.S., and Carew, T.J. 2004b. Neural circuit of tail-elicited siphon withdrawal in Aplysia, I: Differential lateralization of sensitization and dishabituation. J. Neurophysiol. 91: 666-677.

Byrne, J.H., Castellucci, V.F., and Kandel, E.R. 1978. Contribution of individual mechanoreceptor sensory neurons to defensive gill-withdrawal reflex in Aplysia. J. Neurophysiol. 41: 418-431.

Carpenter, D.O., Swann, J.W., and Yarowsky, P.J. 1977. Effect of curare on responses to different putative neurotransmitters in Aplysia neurons. J. Neurobiol. 8: 119-132.

Castellucci, V., Pinsker, H., Kupfermann, I., and Kandel, E.R. 1970. Neuronal mechanisms of habituation and dishabituation of the gill-withdrawal reflex in Aplysia. Science 167: 1745-1748.

Castellucci, V.F., Carew, T.J., and Kandel, E.R. 1978. Cellular analysis of long-term habituation of the gill-withdrawal reflex of Aplysia californica. Science 202: 1306-1308.

Christoffersen, G.R.J. 1997. Habituation: Events in the history of its characterization and linkage to synaptic depression: A new proposed kinetic criterion for its identification. Prog. Neurobiol. 53: 45-66.

Cleary, L.J. and Byrne, J.H. 1993. Identification and characterization of a multifunctional neuron contributing to defensive arousal in Aplysia. J. Neurophysiol. 70: 1767-1776.

Cleary, L.J., Byrne, J.H., and Frost, W.N. 1995. Role of interneurons in defensive reflexes in Aplysia. Learn. Mem. 2: 133-151.

Cohen, T.E., Kaplan, S.W., Kandel, E.R., and Hawkins, R.D. 1997. A simplified preparation for relating cellular events to behavior: Mechanisms contributing to habituation, dishabituation, and sensitization of the Aplysia gill-withdrawal reflex. J. Neurosci. 17: 2886-2899.

Diaz-Rios, M., Suess, E., and Miller, M.W. 1999. Localization of GABA-like immunoreactivity in the central nervous system of Aplysia californica. J. Comp. Neurol. 413: 255-270.

Ezzeddine, Y. and Glanzman, D.L. 2003. Prolonged habituation of the gill-withdrawal reflex in Aplysia depends on protein synthesis, protein phosphatase activity, and postsynaptic glutamate receptors. J. Neurosci. 23: 9585-9594.

Fischer, T.M. and Carew, T.J. 1993. Activity-dependent potentiation of recurrent inhibition: A mechanism for dynamic gain control in the siphon withdrawal reflex of Aplysia. J. Neurosci. 13: 1302-1314.

. 1995. Cutaneous activation of the inhibitory L30 interneurons provides a mechanism for regulating adaptive gain control in the siphon withdrawal reflex in Aplysia. J. Neurosci. 15: 762-773.

Frost, W.N. and Kandel, E.R. 1995. Structure of the network mediating siphon-elicited siphon withdrawal in Aplysia. J. Neurophysiol. 73: 
2413-2427.

Frost, L., Kaplan, S.W., Cohen, T.E., Henzi, V., Kandel, E.R., and Hawkins, R.D. 1997. A simplified preparation for relating cellular events to behavior: Contributions of LE and unidentified siphon sensory neurons to mediation and habituation of the Aplysia gilland siphon-withdrawal reflex. J. Neurosci. 17: 2900-2913.

Giller Jr., E. and Schwartz, J.H. 1971a. Acetylcholinesterase in identified neurons of abdominal ganglion of Aplysia californica. J. Neurophysiol. 34: $108-115$.

. 1971b. Choline acetyltransferase in identified neurons of abdominal ganglion of Aplysia californica. J. Neurophysiol. 34: 93-107.

Glanzman, D.L., Groves, P.M., and Thompson, R.F. 1972. Stimulus generalization of habituation in spinal interneurons. Physiol. Behav. 8: $155-158$.

Groves, P.M. and Thompson, R.F. 1970. Habituation: A dual process theory. Psych. Rev. 77: 419-450.

Harris, J.D. 1943. Studies of non-associative factors inherent in conditioning. Comp. Psych. Monogr. 18: 74

Hawkins, R.D. 1981. Interneurons involved in mediation and modulation of gill-withdrawal reflex in Aplysia, III: Identified facilitating neurons increase $\mathrm{Ca} 2+$ currents in sensory neurons. $J$. Neurophysiol. 45: 327-339.

Hawkins, R.D., Castellucci, V.F., and Kandel, E.R. 1981. Interneurons involved in mediation and modulation of gill-withdrawal reflex in Aplysia, I: Identification and characterization. J. Neurophysiol. 45: 304-314.

Hickie, C. and Walters, E.T. 1995. Motor neuronal control of tail-directed and head-directed siphon responses in Aplysia californica. J. Neurophysiol. 74: 307-321.

Horn, G. 1967. Neuronal mechanisms of habituation. Nature 215: 707 711 .

Humphrey, G. 1933. The nature of learning. Kegan Paul, Trench, Trubner \& Co., London.

Jing, J., Vilim, F.S., Wu, J.S., Park, J.H., and Weiss, K.R. 2003. Concerted GABAergic actions of Aplysia feeding interneurons in motor program specification. J. Neurosci. 23: 5283-5294.

Kandel, E.R. 1979.Behavioral biology of Aplysia. Freeman, San Francisco, CA.

Kehoe, J. 1972. Three acetylcholine receptors in Aplysia neurones. J. Physiol. (Lond.) 225: 115-146.

Koester, J. and Kandel, E.R. 1977. Further identification of neurons in the abdominal ganglion of Aplysia using behavioral criteria. Brain Res. 121: 1-20.

Krasne, F.B. 1969. Excitation and habituation of the crayfish escape reflex: The depolarizing response in lateral giant fibers of the isolated abdomen. J. Exp. Biol. 50: 29-46.

Krasne, F.B. and Teshiba, T.M. 1995. Habituation of an invertebrate escape reflex due to modulation by higher centers rather than local events. Proc. Natl. Acad. Sci. 92: 3362-3366.

Kupfermann, I., Castellucci, V., Pinsker, H., and Kandel, E. 1970. Neuronal correlates of habituation and dishabituation of the gill-withdrawal reflex in Aplysia. Science 167: 1740-1748.

Lukowiak, K. 1978. Picrotoxin prevents habituation of the gill withdrawal reflex in Aplysia. Can. I. Physiol. Pharm. 56: 1079-1082.

Marinesco, S. and Carew, T.J. 2002. Serotonin release evoked by tail nerve stimulation in the central nervous system of Aplysia: Characterization and relationship to heterosynaptic plasticity. J. Neurosci. 22: 2299-2312.

Pinsker, H., Kupferman, I., Castellucci, V., and Kandel, E.R. 1969. Cellular analysis of behavioral reflex habituation in Aplysia. Fed. Proc. 28: 588.

. 1970. Habituation and dishabituation of gill-withdrawal reflex in Aplysia. Science 167: 1740-1742.

Segal, M. and Koester, J. 1982. Convergent cholinergic neurons produce similar postsynaptic actions in Aplysia: Implications for neural organization. J. Neurophysiol. 47: 742-759.

Srivatsan, M. and Peretz, B. 1996. Effect of acetylcholinesterase inhibition on behavior is age-dependent in freely moving Aplysia. Behav. Brain Res. 77: 115-124.

Stopfer, M. and Carew, T.J. 1996. Heterosynaptic facilitation of tail sensory neuron synaptic transmission during habituation in tail-induced tail and siphon withdrawal reflexes of Aplysia. J. Neurosci. 16: 4933-4948.

Tauc, L. and Gerschfeld, H.M. 1962. A cholinergic mechanism of inhibitory synaptic transmission in a molluscan nervous system. $J$. Neurophysiol. 25: 236-262.

Thompson, R.F. and Spencer, W.A. 1966. Habituation: A model phenomenon for the study of neuronal substrates of behavior. Psych. Rev. 73: 16-43.

Thorpe, W.H. 1963. Learning and instinct in animals. Methuen, London.

Trudeau, L-E. and Castellucci, V.F. 1993. Functional uncoupling of inhibitory interneurons plays an important role in short-term sensitization of Aplysia gill and siphon withdrawal reflex. J. Neurosci. 13: 2126-2135.

Walters, E.T. 1987. Multiple sensory neuronal correlates of site-specific sensitization in Aplysia. J. Neurosci. 7: 408-417.

Walters, E.T., Byrne, J.H., Carew, T.J., and Kandel, E.R. 1983. Mechanoafferent neurons innervating tail of Aplysia, I: Response properties and synaptic connections. J. Neurophysiol. 50: 1522-1542.

Wright, W.G. and Carew, T.J. 1995. A single identified interneuron gates tail-shock induced inhibition in the siphon withdrawal reflex of Aplysia. J. Neurosci. 15: 790-797.

Zhang, H., Wainwright, M., Byrne, J.H., and Cleary, L.J. 2003. Quantitation of contacts among sensory, motor, and serotonergic neurons in the pedal ganglion of Aplysia. Learn. Mem. 10: 387-393.

Zucker, R.S. 1972. Crayfish escape behavior and central synapses, II: Physiological mechanisms underlying behavioral habituation. J. Neurophysiol. 35: 621-637.

Received July 8, 2004; accepted in revised form November 10, 2004.

\section{Learning \& Memory}




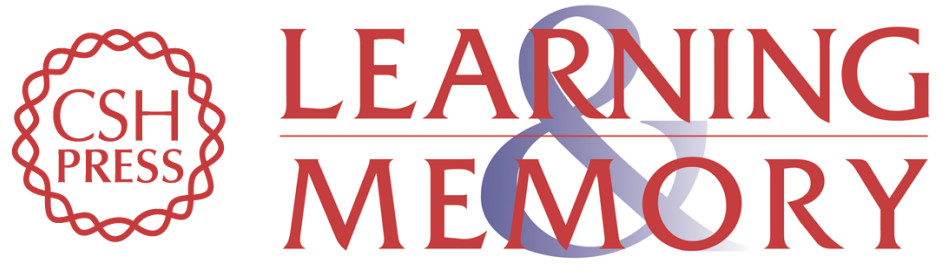

\section{Differential role of inhibition in habituation of two independent afferent pathways to a common motor output}

Adam S. Bristol and Thomas J. Carew

Learn. Mem. 2005, 12:

Access the most recent version at doi:10.1101/lm.83405

References This article cites 49 articles, 21 of which can be accessed free at: http://learnmem.cshlp.org/content/12/1/52.full.html\#ref-list-1

License

Email Alerting Receive free email alerts when new articles cite this article - sign up in the box at the Service top right corner of the article or click here. 